\title{
Transport and optical properties of single- and bilayer black phosphorus with defects
}

\author{
Shengjun Yuan, ${ }^{*}$ A. N. Rudenko, and M. I. Katsnelson \\ Radboud University, Institute for Molecules and Materials, Heijendaalseweg 135, 6525 AJ Nijmegen, The Netherlands
}

(Received 9 November 2014; revised manuscript received 12 March 2015; published 27 March 2015)

\begin{abstract}
We study the electronic and optical properties of single- and bilayer black phosphorus with short- and long-range defects by using the tight-binding propagation method. Both types of defect states are localized and induce a strong scattering of conduction states, reducing significantly the charge carrier mobility. In contrast to properties of pristine samples, the anisotropy of defect-induced optical excitations is suppressed due to the isotropic nature of the defects. We also investigate the Landau level spectrum and magneto-optical conductivity and find that the discrete Landau levels are sublinearly dependent on the magnetic field and energy level index, even at low defect concentrations.
\end{abstract}

DOI: 10.1103/PhysRevB.91.115436

\section{INTRODUCTION}

Black phosphorus (BP) is a layer material in which the atomic layers are coupled by weak van der Waals interactions. Few-layer BP is a new kind of two-dimensional (2D) material that can be obtained by a mechanical exfoliation method from BP films [1-6], a common fabricating method of producing 2D materials. BP is a semiconductor with a layer-dependent direct band gap, which is crucial for a number of applications such as field-effect transistors [7-11]. The anisotropic optical response of BP [3,7,9,12-15], which is not typical for other known 2D materials, makes it an ideal material for a photon polarizer. On the other hand, unlike graphene and transition-metal dichalcogenides, which are chemically stable under ambient conditions, BP samples are shown to be very sensitive to the environment $[4,5,16-18]$. This is due to the high reactivity of BP with respect to air and might limit their application in real devices. In this regard, the role of defects and impurities in BP represents an important issue with theoretical and practical relevance.

In 2D materials, the scattering induced by short-range point defects (like adsorbates in graphene and sulfur vacancies in $\mathrm{MoS}_{2}$ ) are shown to be one of the main mechanisms dominating the charge mobility [19-26]. The point defects are the so-called resonant scatterers because they can provide resonances (quasilocalized states) near the neutrality point (in graphene [22,23]) or within the band gap (in the semiconducting transition-metal dichalcogenides such as $\mathrm{MoS}_{2}$ and $\mathrm{WS}_{2}$ $[25,26])$. The emergence of the midgap states due to point defects in BP has been observed in several first-principles calculations, such as single vacancy $[27,28]$, substitutional $p$ dopants (Te, C) [27], oxygen bridge-type defects [29], and absorption of organic molecules [30] or adatoms ( $\mathrm{Si}, \mathrm{Ge}, \mathrm{Au}$, Ti, V) [31]. But it remains unclear what is the influence of the resonant point defects to the transport and optical properties of BP, because the first-principles calculations are limited by the sample size that is computationally too expensive to consider a large sample with many defects. Another typical disorder in 2D materials is the long-range electron-hole puddles [19-21], which are inhomogeneities of carrier density and have been observed experimentally $[19,32]$. The origin of electron-hole puddles could be charged impurities and defects located on the substrate [33-35] or surface corrugations such

\footnotetext{
*s.yuan@science.ru.nl
}

PACS number(s): 73.22.-f, 61.43.Bn, 71.23.-k, 78.20.Bh

as ripples and wrinkles [36,37]. Unlike the point defects, the electron-hole puddles do not introduce strong resonances in the spectrum and therefore are referred to as typical nonresonant defects. In this paper, the study of disordered samples are performed by using the tight-binding propagation method (TBPM) $[22,23,38,39]$, which is extremely efficient for large-scale calculation of systems with more than millions of atoms.

\section{TIGHT-BINDING MODEL}

The TB Hamiltonian for pristine BP is based on the $G W$ approximation and is given as follows [8]:

$$
H=\sum_{i} \varepsilon_{i} n_{i}+\sum_{i \neq j} t_{i j} c_{i}^{\dagger} c_{j}+\sum_{i \neq j} t_{i j}^{\perp} c_{i}^{\dagger} c_{j},
$$

where the summation runs over the lattice sites of single- or bilayer BP, $\varepsilon_{i}$ is the energy of the electron at site $i, t_{i j}^{(\perp)}$ is the intralayer (interlayer) hopping parameter between the $i$ th and $j$ th sites, and $c_{i}^{\dagger}\left(c_{j}\right)$ is the creation (annihilation) operator of electrons at site $i(j)$. The parameters $t_{i j}, t_{i j}^{\perp}$, and $\epsilon_{i}$ were obtained on the basis of accurate $a b$ initio calculations within the $G_{0} W_{0}$ approximation by mapping the entire manifold of $s p$ states onto the minimal set (one site per $\mathrm{P}$ atom) of relevant states near the band gap. Specifically, we use five intralayer $\left(t_{1}=-1.220 \mathrm{eV}, t_{2}=3.665 \mathrm{eV}, t_{3}=-0.205 \mathrm{eV}\right.$, $\left.t_{4}=-0.105 \mathrm{eV}, t_{5}=-0.055 \mathrm{eV}\right)$ and four interlayer hoppings $\left(t_{1}^{\perp}=0.295 \mathrm{eV}, t_{2}^{\perp}=0.273 \mathrm{eV}, t_{3}^{\perp}=-1.151 \mathrm{eV}\right.$, $t_{4}^{\perp}=-0.091 \mathrm{eV}$ ), which is schematically shown in Fig. 1, and an energy splitting of $\Delta \epsilon=1.0 \mathrm{eV}$ between the nonequivalent electrons in bilayer BP [8]. The resulting TB model accurately describes the quasiparticle electron and hole bands of single-layer and bilayer BP in the range of $\sim 0.3 \mathrm{eV}$ beyond the gap [8].

The energy dispersions $E\left(k_{x}, k_{y}\right)$ can be obtained analytically by diagonalizing the TB Hamiltonian (see the band structure plotted in Fig. 1 and detailed calculations in the appendix). The anisotropy can be further identified directly from the anisotropic Fermi velocities and effective masses shown in Fig. 2. The Fermi velocity $v_{\alpha}=\frac{1}{\hbar} \frac{\partial E}{\partial k_{\alpha}}$ and effective mass $m_{\alpha}=\hbar^{2} / \frac{\partial^{2} E}{\partial k_{\alpha}^{2}}$ are calculated from the energy dispersion relations (see details in the appendix). The velocity of an electron (hole) along the armchair direction is much larger than the value along the zigzag direction, with both linear 

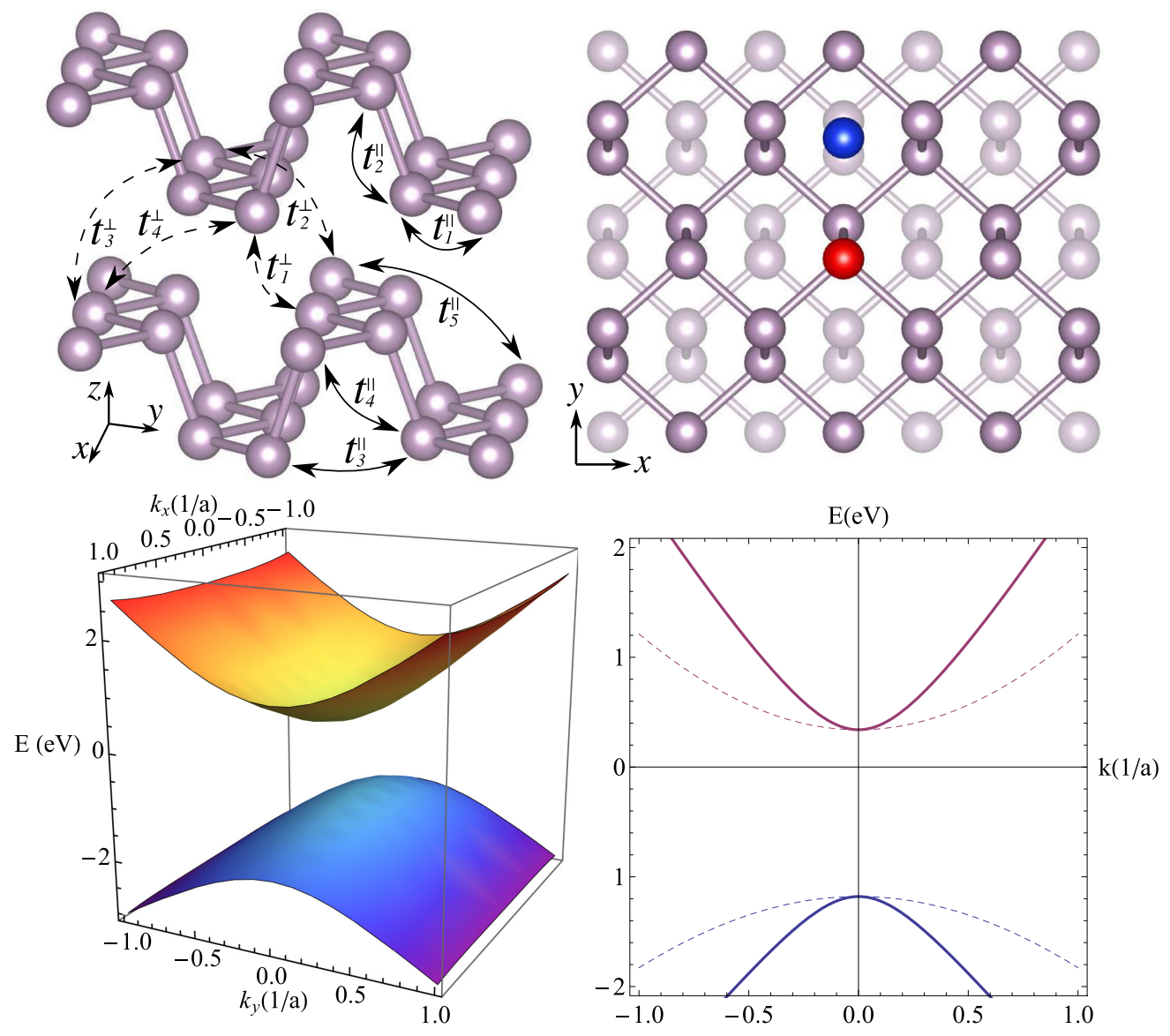

FIG. 1. (Color online) (top left) Schematic representation of the atomic structure and hopping parameters of the TB model of BP. (top right) Top view of the atomic structure; the red dot represents a point defect, and the blue dot represents a long-range defect at the center of the projected honeycomb lattice on the surface. (bottom left) Three-dimensional contour plot of the lowest valence and conduction bands. (bottom right) Lowest valence and conduction bands along armchair (solid lines) and zigzag (dashed lines) directions.
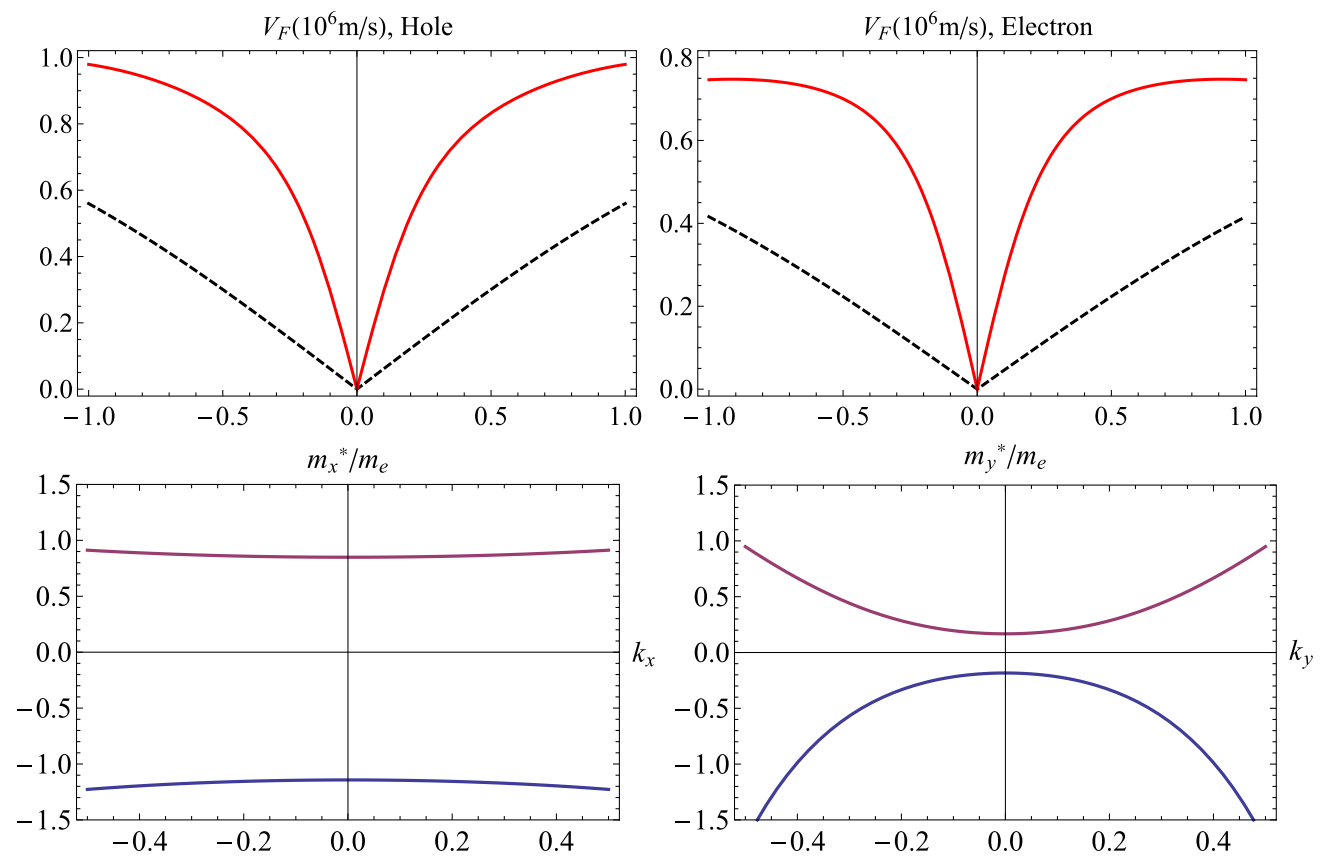

FIG. 2. (Color online) (top) Electron and hole velocities in single-layer BP as a function of wave vector along armchair (red solid line) and zigzag (black dashed line) directions. (bottom) Effective mass along armchair $(Y)$ and zigzag $(X)$ directions. The wave vector $k$ is in units of $1 / a$, where $a \approx 2.216 \AA$ is the atomic distance between two nearest neighbors. 
$k$-dependent velocities around the $\Gamma$ point $(k=0)$. This is different from the Dirac fermion in graphene which travels with constant velocity $v_{F} \simeq c / 300$, where $c$ is the speed of light in vacuum. The effective masses at the $\Gamma$ point along the armchair direction are $m_{y}^{v}=0.184 m_{e}$ for hole, and $m_{y}^{c}=$ $0.167 m_{e}$ for electron. Here, $m_{e}$ is the free electron mass. The effective masses along the zigzag direction are much heavier: $m_{x}^{v}=1.143 m_{e}$ for hole and $m_{x}^{c}=0.849 m_{e}$ for electron.

\section{TIGHT-BINDING PROPAGATION METHOD}

The electronic and optical properties of single- and bilayer $\mathrm{BP}$ are calculated by using the TBPM [22,23,39,40], in which the initial state $|\varphi\rangle$ is chosen as a random superposition of all sites over the whole space which covers all the energy eigenstates $[23,40]|\varphi\rangle=\sum_{i} a_{i}|i\rangle$, where $a_{i}$ are random complex numbers normalized as $\sum_{i}\left|a_{i}\right|^{2}=1$, and $|i\rangle$ represents a basis state at site $i$. The density of states can be obtained by Fourier transformation of the overlap between the time-evolved state $|\varphi(t)\rangle \equiv e^{-i \mathcal{H} t}|\varphi\rangle$ and the initial state $|\varphi\rangle$ as $[23,40]$

$$
\rho(\varepsilon)=\frac{1}{2 \pi} \int_{-\infty}^{\infty} e^{i \varepsilon t}\langle\varphi \mid \varphi(t)\rangle d t .
$$

Here we use units such that $\hbar=1$. The time-evolution operator $e^{-i \mathcal{H} t}$ is calculated numerically by using the Chebyshev polynomial algorithm, which is extremely efficient for a TB Hamiltonian $\mathcal{H}$ which is a sparse matrix. Within the TBPM, the optical conductivity (omitting the Drude contribution at $\omega=0$ ) is calculated by using the Kubo formula $[23,42]$

$$
\begin{aligned}
\sigma_{\alpha \beta}(\omega)= & \lim _{\epsilon \rightarrow 0^{+}} \frac{e^{-\tilde{\beta} \omega}-1}{\omega \Omega} \int_{0}^{\infty} e^{-\epsilon t} \sin \omega t \\
& \times 2 \operatorname{Im}\left\langle\varphi\left|f(\mathcal{H}) J_{\alpha}(t)[1-f(\mathcal{H})] J_{\beta}\right| \varphi\right\rangle d t,
\end{aligned}
$$

where $\tilde{\beta}=1 / k_{B} T$ is the inverse temperature, $\Omega$ is the sample area, $f(\mathcal{H})=1 /\left[e^{\tilde{\beta}(\mathcal{H}-\mu)}+1\right]$ is the Fermi-Dirac distribution operator, and the time-dependent current operator in the $\alpha$ $(=x$ or $y)$ direction is defined as $J_{\alpha}(t)=e^{i \mathcal{H} t} J_{\alpha} e^{-i \mathcal{H} t}$.

The optical conductivity at an arbitrary direction is

$$
\sigma_{\theta}(\omega)=\sigma_{x x}(\omega) \cos ^{2} \theta+\sigma_{y y}(\omega) \sin ^{2} \theta,
$$

where $\theta$ is the angle between the polarized direction and $x$ axis. Equation (4) can be derived from the Kubo formula by using the relation of the current operator $J_{\theta}=J_{x} \cos \theta+J_{y} \sin \theta$.

The reflection and transmission of a polarized light through a BP film can be solved by using the Maxwell equations with a conducting layer. For the case of normal incidence, the reflectivity can be expressed as [41]

$$
r_{\theta}(\omega)=-\frac{\varepsilon_{0} c\left(\sqrt{\varepsilon_{2}}-\sqrt{\varepsilon_{1}}\right)+\sigma_{\theta}(\omega)}{\varepsilon_{0} c\left(\sqrt{\varepsilon_{2}}+\sqrt{\varepsilon_{1}}\right)+\sigma_{\theta}(\omega)},
$$

where $\varepsilon_{0}$ is the permittivity of vacuum, $\varepsilon_{1}$ and $\varepsilon_{2}$ the relative permittivity of two media on the two sides of BP film, and $c$ is the speed of light. The reflection and transmission probabilities are given by $\mathcal{R} \approx|r|^{2}$ and $\mathcal{T} \approx|1+r|^{2} \sqrt{\varepsilon_{2} / \varepsilon_{1}}$, and the absorption coefficient is $\alpha=1-\mathcal{R}-\mathcal{T}$. The absorption coefficient of BP films can be obtained directly from optical measurements, such as Fourier transform infrared spectroscopy (FTIS)[3,13]. In this work, we show the results with $\varepsilon_{1}=\varepsilon_{2}=1$ in order to ignore the influence of the dielectric substrate.

The dc conductivity at energy $E=\varepsilon$ is calculated by using the Kubo formula at $\omega \rightarrow 0[23,42]$

$$
\begin{aligned}
\sigma_{\alpha \alpha} & =\lim _{\tau \rightarrow \infty} \sigma_{\alpha \alpha}(\tau) \\
& =\lim _{\tau \rightarrow \infty} \frac{\rho(\varepsilon)}{\Omega} \int_{0}^{\tau} d t \operatorname{Re}\left[e^{-i \epsilon t}\left\langle\varphi\left|J_{\alpha} e^{i \mathcal{H} t} J_{\alpha}\right| \varepsilon\right\rangle\right],
\end{aligned}
$$

where $|\varepsilon\rangle$ is the normalized quasi-eigenstate [23,38,39]. The semiclassic dc conductivity $\sigma_{s c}$ without considering the effect of Anderson localization is defined as the maximum of $\sigma_{\alpha \alpha}(\tau)$ obtained from the integral in Eq. (6). The measured field-effect carrier mobility is related to the semiclassic dc conductivity as $\mu(E)=\sigma_{s c}(E) / e n_{e}(E)$, where the carrier density $n_{e}(E)$ is obtained from the integral of density of states via $n_{e}(E)=$ $\int_{0}^{E} \rho(\varepsilon) d \varepsilon$.

We use periodic boundary condition in our calculations, and the system size is fixed as $4096 \times 4096$ for single-layer BP and $2048 \times 2048$ for bilayer BP.

\section{MODEL OF DEFECTS}

In the employed TB model, the point defects are modeled by elimination of atoms randomly over the whole sample, which can be viewed as phosphorus single vacancies, chemical adsorbates such as covalently bonded adatoms or admolecules, or substitution of other types of atoms which prevent the electronic hopping to their neighbors [19-26]. The amount of point defects is described by $n_{x}$, which is the probability for a single defect to appear at one lattice site. The electron-hole puddles, in which the spatial charge inhomogeneity leads to a local change of onsite potentials, can be represented as a correlated Gaussian potential in the TB model $[24,38,43]$. The value of the potential at site $i$ follows

$$
v_{i}=\sum_{k=1}^{N_{\text {imp }}^{v}} U_{k} \exp \left(-\frac{\left|\mathbf{r}_{i}-\mathbf{r}_{k}\right|^{2}}{2 d^{2}}\right),
$$

where $N_{\text {imp }}^{v}$ is the number of the Gaussian centers, $\mathbf{r}_{k}$ is the position of the $k$ th Gaussian center, which are chosen to be randomly distributed over the centers of the projected lattice on the surface, $U_{k}$ is the amplitude of the potential at the Gaussian center, which is uniformly random in the range $[-\Delta, \Delta]$, and $d$ is interpreted as the effective potential radius. The typical values of $d$ used in our model are $\Delta=5 \mathrm{eV}$ and $d=5 a$ for electron-hole puddles [24,38]. Similarly, the amount of electron-hole puddles is measured by $n_{c}$, which is the probability for a Gaussian potential to appear.

In order to investigate the influence of the defects on the electronic structure of BP, we calculate the density of states (DOS) of samples with randomly distributed point defects or Gaussian potentials. As we expect, the presence of point defects leads to midgap states within the energy band gap, which can be identified by the sharp peaks in the DOS shown in Figs. 3(a) and 3(b), where the number of defect states is proportional to the concentration of point defects $[23,26]$. Similar midgap states appear in the first-principles calculations of BP with different types of point defects [27-31]. On the 

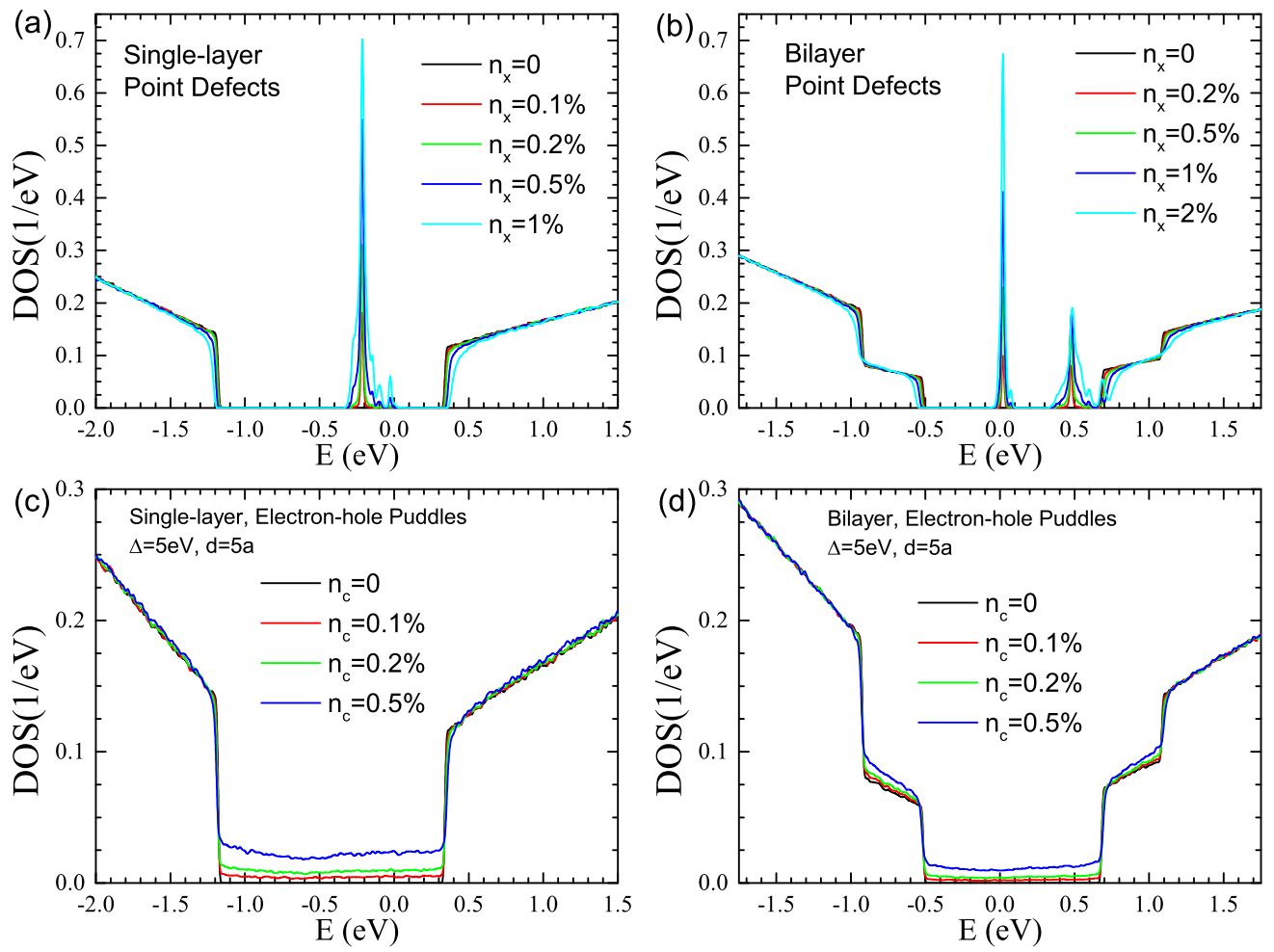

FIG. 3. (Color online) Density of states of single-layer (left columns) and bilayer (right columns) with point defects (upper rows) or electron-hole puddles (bottom rows). The value $n_{x}\left(n_{c}\right)=0.1 \%$ corresponds to defect a concentration of $2.98(1.49) \times 10^{12}$ per $\mathrm{cm}^{2}$.

other hand, the presence of electron-hole puddles does not introduce any resonant states [see Figs. 3(c) and 3(d)]. Instead, there is a uniform enhancement of the DOS within the gap due to the random distribution of positive and negative potentials, whereas the increased amplitude is proportional to the number (concentration) of potential puddles.

\section{TRANSPORT PROPERTIES}

The calculations of transport properties by using the Kubo formula show that for both point defects and electron-hole puddles, the impurity states within the band gap are insulating states (see Fig. 4). This is due to the Anderson localization in disordered 2D systems [44,45], and the result is not sensitive to the defect concentration. On the other hand, the dc conductivities in the valence and conduction bands decrease monotonically as the defect concentration increases. This is consistent with the qualitative analysis by using the $T$ matrix [26,46]. For example, point defects in our model are represented as impurities with infinite onsite potential, and the scattering by these impurities leads to $T(E) \rightarrow-1 / g_{0}(E)$, where $g_{0}(E)$ is the local unperturbed Green's function.
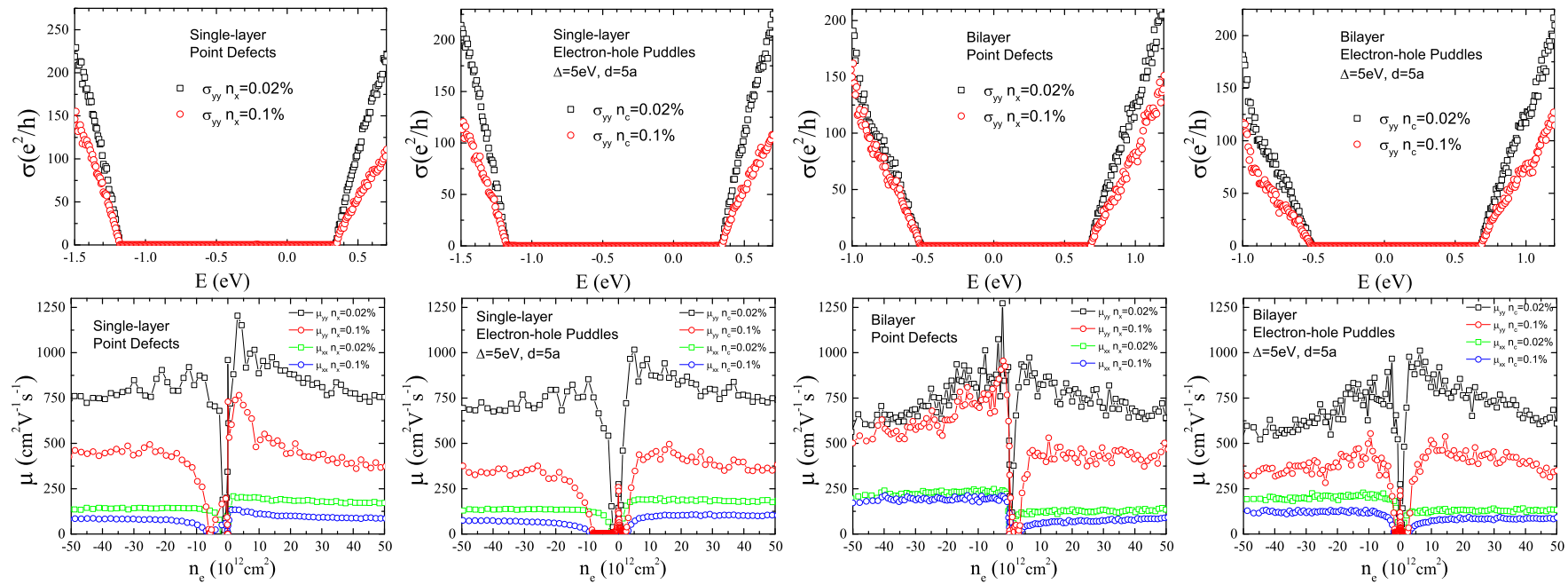

FIG. 4. (Color online) Transport properties of single- and bilayer with defects. (top) de conductivity as a function of doping energy. (bottom) carrier mobility as a function of carrier density. 
For a semiconductor with electron-hole asymmetry like BP, the density of states follows approximately $N_{0}(E)=D_{c} \Theta$ $\left(E-E_{c}\right)+D_{v} \Theta\left(E_{v}-E\right)$, where $E_{c(v)}$ is the energy at the edge of conduction (valence) band. The local unperturbed Green's function is $[26,46]$

$$
g_{0}(E)=D_{c} \ln \left|\frac{E-E_{c}}{E-W_{c}}\right|+D_{v} \ln \left|\frac{E+W_{v}}{E-E_{v}}\right|,
$$

where $W_{c(v)}$ is the width of the conduction (valence) bands. The dc conductivity is proportional to the inverse of the defect concentration $n_{x}$, as it can be expressed as $\sigma=\left(2 e^{2} / h\right) E \tau$ where $\tau^{-1}=(2 \pi / \hbar) n_{x}|T(E)|^{2} N_{0}(E)$ is the scattering rate in terms of $n_{x}$

Furthermore, the carrier-density dependence of the mobility shows different electron-hole asymmetry in singleand bilayers. The electron mobility is higher than the hole mobility in single-layer material, but it is opposite in the bilayer material. For example, for single layer with a defect concentration $n_{x}=0.02 \%$, the electron mobility along the armchair direction is about $1200 \mathrm{~cm}^{2} \mathrm{~V}^{-1} \mathrm{~s}^{-1}$ at a carrier density $n_{e}=5 \times 10^{12} \mathrm{~cm}^{2}$, which is larger than the hole mobility $\left(700 \mathrm{~cm}^{2} \mathrm{~V}^{-1} \mathrm{~s}^{-1}\right)$ at the same order of carrier density. The asymmetry of the mobility becomes more obvious with increased number of defects. For the same carrier density considered above, when the defect concentration increases to $n_{x}=0.1 \%$, the mobility drops to $700 \mathrm{~cm}^{2} \mathrm{~V}^{-1} \mathrm{~s}^{-1}$ for electrons and less than $50 \mathrm{~cm}^{2} \mathrm{~V}^{-1} \mathrm{~s}^{-1}$ for holes. On the contrary, for bilayer with $n_{x}=0.1 \%$, the electron mobility is about $300 \mathrm{~cm}^{2} \mathrm{~V}^{-1} \mathrm{~s}^{-1}$ at the carrier density $n_{e}=5 \times$ $10^{12} \mathrm{~cm}^{2}$, much smaller than the corresponding hole mobility $\left(800 \mathrm{~cm}^{2} \mathrm{~V}^{-1} \mathrm{~s}^{-1}\right)$. The different electron and hole mobility suggests that single layer is more suitable for the application as an $n$-doped field-effect transistor, while bilayer is better as a $p$-doped field-effect transistor. The drain current modulation of $n$-doped single layer and $p$-doped bilayer can reach the experimental observed value $\left(\sim 10^{5}\right.$ in Ref. [1]) even with a defect concentration $n_{x}\left(n_{c}\right)=0.1 \%$.

\section{OPTICAL PROPERTIES}

Further calculations of the optical conductivity show that there will be extra excitations below the pristine optical gap along both armchair and zigzag directions (see Fig. 5), due to the presence of defect states. Using the presence of point defects as an example, for single layer, the excitations between the midgap states at $E \approx-0.2 \mathrm{eV}$ and the states at the conduction band edge $(E \approx 0.3 \mathrm{eV})$ reduce the optical gap to $0.5 \mathrm{eV}$, much smaller than the value $(1.4 \mathrm{eV})$ in the pristine sample. For bilayer, there are two groups of defect states,
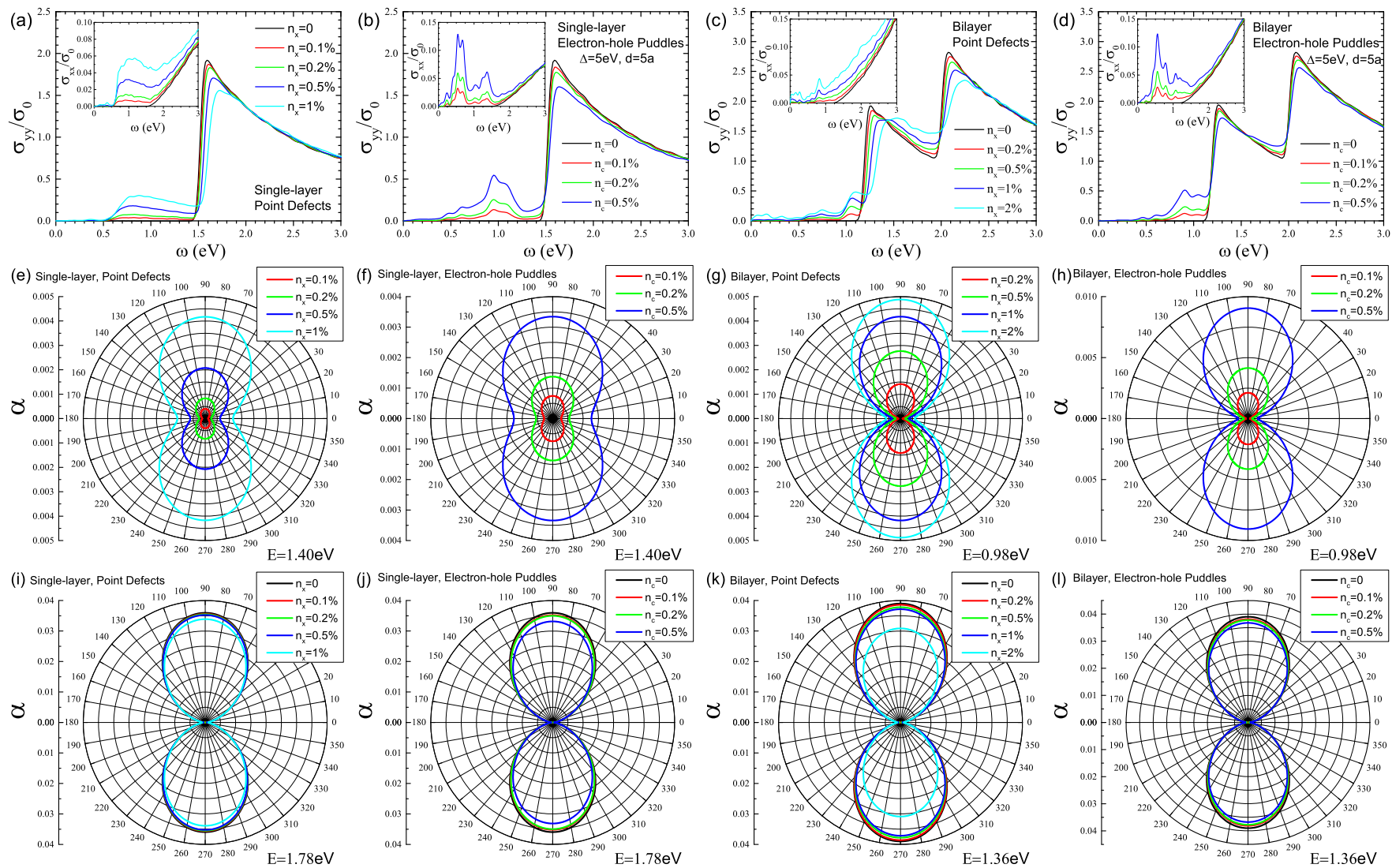

FIG. 5. (Color online) Optical conductivity as (a)-(d) a function of energy and optical absorption and (e)-(l) as a function of polarized angle for suspended single- and bilayer BP with defects. The energies of the photon for single-layer are (e), (f) $1.4 \mathrm{eV}$ and (i), (j) $1.78 \mathrm{eV}$, and for bilayer are (j), (k) $0.98 \mathrm{eV}$ and (h), (l) $1.36 \mathrm{eV}$. The black lines are the results for pristine samples, and the colored lines are the results for disordered samples with different concentrations of defects. Throughout this work, we fix the temperature to $T=300 \mathrm{~K}$ and the chemical potential to $\mu_{F}=0$ for the optical conductivity and normalize the conductivity to $\sigma_{0}=\pi e^{2} / 2 h$, the universal optical conductivity of single-layer graphene in the visible-light region. 
e.g., one sharp peak at $E \approx 0.02 \mathrm{eV}$ and another broader peak at $E \approx 0.48 \mathrm{eV}$ [Fig. 3(b)]. The reduced optical gap $(\sim 0.52 \mathrm{eV})$ is due to the excitations between the valence band edge $(-0.5 \mathrm{eV})$ and the conduction peak at $E \approx 0.02 \mathrm{eV}$. Another effect due to the appearance of the defects is the smearing of the optical peaks along the armchair direction, i.e., the peak at $\omega \approx 1.5 \mathrm{eV}$ for single- and $\omega \approx 1.2 \mathrm{eV}$ for bilayer as can be seen from Fig. 5. It is important to note that, although there are changes of the optical conductivities along both armchair and zigzag directions, the optical spectrum has different anisotropy below and above the band gap. The angle-dependent absorption coefficients of linearly polarized light in Fig. 5 show that the anisotropy remains unchanged for photons with energy higher than the gap width, but becomes much weaker within the gap. That is, in the presence of defects, the transport along the armchair direction is still much stronger than the zigzag direction above the gap but becomes comparable within the gap. The difference of the anisotropy is more clear in the case of single layer.

The influence of defects on the anisotropy of optical properties can be explained by the isotropic nature of the defect Hamiltonian. A point-like resonant defect is equivalent to a single lattice site with strong on-site potential or out-of-plane hopping, and for a nonresonant defect with a real-space Gaussian profile, the value of the potential only depends on the relative distance to the Gaussian center. That is, the extra Hamiltonian terms introduced by both types of defects are isotropic. Therefore, the optical excitations involving the defect states become less anisotropic compared to excitations in pristine BP. Furthermore, because the new excitation is proportional to the number of defect states, we expect that (1) the increase of the defect concentration will enhance the excitation below the optical gap, and (2) the profile of the angle-dependent optical spectroscopy should be robust against the defect concentration, because the defect states are localized and separated according to the transport calculations. These conclusions are confirmed by the optical spectroscopy shown in Fig. 5.

The restrain of the anisotropy obtained in our calculations is similar to that observed in recent excitation measurements of few-layer BP films [3]. The difference is that the experiment is performed on a BP film with a much smaller band gap (about $0.3 \mathrm{eV}$ ) comparing to these in single-layer $(1.5 \mathrm{eV})$ and bilayer $(1.2 \mathrm{eV})$. As the band gap in multilayer BP is highly reduced, the impurity band(s) due to the presence of defects could have overlaps with the pristine bands, which will restrain the anisotropy even above the optical gap. We leave out the study of disordered multilayer BP for future work with a further development of TB models.

\section{LANDAU LEVEL SPECTRUM AND MAGNETO-OPTICAL SPECTROSCOPY}

In the presence of a perpendicular magnetic field $B$, the quantization of the energy levels leads to separated Landau levels (LLs). The low-energy physics of single-layer BPs can be described by an effective $k \cdot p$ model $[47,48]$, and the LLs are [49]

$$
E_{n, s}^{k p}=E_{s}+\frac{s e B \hbar}{m_{e}}\left(n+\frac{1}{2}\right) w_{s},
$$

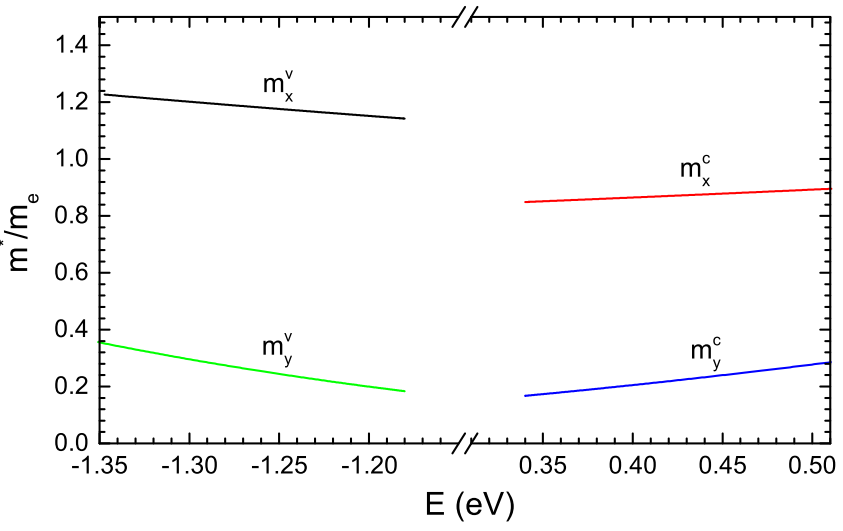

FIG. 6. (Color online) The energy dependence of the anisotropic effective masses of single-layer BP in the TB model.

where $s= \pm 1$ denotes the conduction and valence bands, $E_{+/-}=E_{c / v}$ is the energy at the conduction and valence edge, $n$ is the energy index and $w_{+/-}=m_{e} /\left(m_{x}^{c / v} m_{y}^{c / v}\right)^{1 / 2}$ $\left(m_{x}^{c / v}\right.$ and $m_{y}^{c / v}$ are anisotropic effective masses at the $\Gamma$ point). However, because the $k \cdot p$ model does not capture the energy-dependence of the effective masses (see the results obtained from the TB calculations in Fig. 6), Eq. (9) is not valid at high magnetic field. In fact, as can be seen from Fig. 7, the Landau spectrum obtained from the TB calculations show that the LLs follow a sublinear dependence on the magnetic
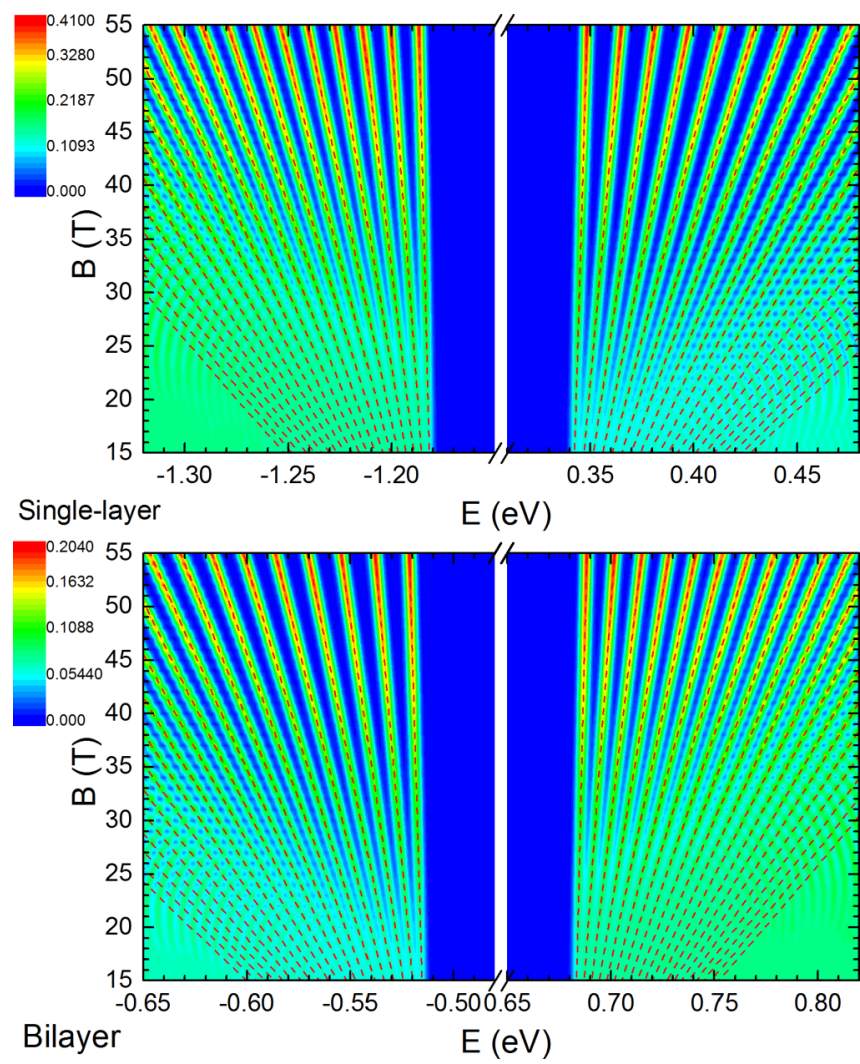

FIG. 7. (Color online) Landau level spectrum of single-layer and bilayer BP in high magnetic field. The red dashed lines are the lowest twenty LLs calculated from Eq. (10). 

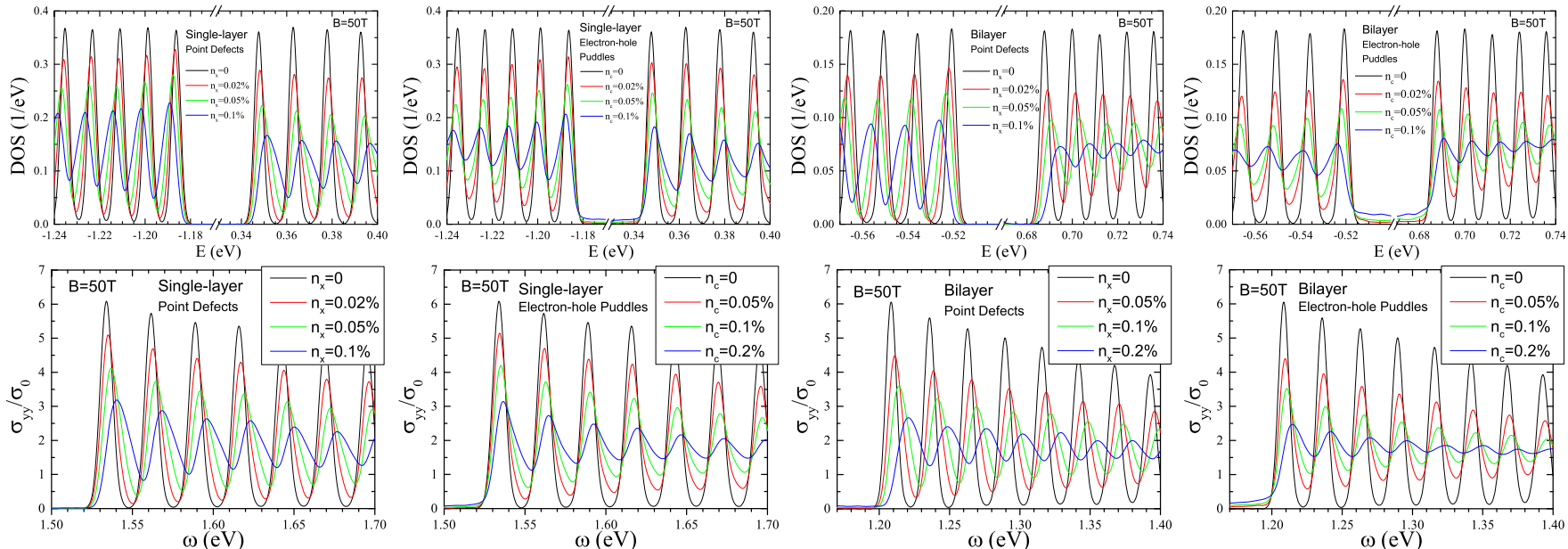

FIG. 8. (Color online) DOS and magneto-optical spectrum of disordered single-layer and bilayer BP with perpendicular magnetic field $B=50 \mathrm{~T}$.

field and energy index $n$, which can be fit as

$$
E_{n, s}=E_{s}+\frac{\operatorname{se} B \hbar}{m_{e}}\left[\left(n+\frac{1}{2}\right) w_{s}-\delta_{s} B n^{p_{s}}\right]
$$

where $E_{+(-)}=0.34 \mathrm{eV}(-1.18 \mathrm{eV}), w_{+(-)}=2.656(2.181)$, $\delta_{+(-)}=0.0005(0.0004)$, and $p_{+/-}=1.8$ for single-layer and $E_{+(-)}=0.6815 \mathrm{eV}(-0.513 \mathrm{eV}), w_{+(-)}=2.14$ (2.67), $\delta_{+(-)}=0.0004(0.00085)$, and $p_{+(-)}=1.8$ (1.73) for bilayer. In the TB calculations, the hopping parameter $t_{m n}$ between two sites is replaced by a Peierls substitution as $t_{m n} \exp \left[\right.$ ie $\left.\int_{m}^{n} \mathbf{A} \cdot d \mathbf{l}\right]$, and we choose the Landau gauge in which the vector potential is $\mathbf{A}=(-B y, 0,0)$. In the presence of either point defects or electron-hole puddles, the LL peaks in the DOS are smeared and suppressed, depending on the defect concentration. The broadening of peaks in the DOS also lead to energy shifts of the LLs; however, for a small concentration of defects, the shifted LLs still follow the sublinear dependence of Eq. (10), but with a smaller $\delta_{s}$. The Landau quantization of energy levels are also observed in the discrete magneto-optical spectrum shown in Fig. 8. The broadening and energy shifts of the optical peaks due to the presence of defects are consistent with the DOS. Here we only present the results along the armchair direction, because the magneto-optical conductivity along the zigzag direction is three-orders of magnitude smaller due to the anisotropy of BP.

\section{CONCLUSION}

In summary, we show that the intrinsic anisotropy of singleand bilayer BP is robust to the presence of defects. The emergence of defect states with short-range point defects is identified by sharp peaks in the DOS within the band gap, which is different from the uniform increase of states with the long-range electron-hole puddles. For both short- and long-range defects, the defect states are insulating due to the Anderson localization in disordered 2D systems, but they cause extra excitations within the optical gap. The dc conductivity as well as carrier mobility beyond the gap are significantly reduced due to the scattering from the defects. The angledependent absorption coefficients of linearly polarized light show that the anisotropy above the band gap is robust against the disorder, but the anisotropy of the new excitations involving the defect states is suppressed because of the isotropic nature of the defects. By fitting the numerical results of the DOS obtained in the TB model, we find a sublinear dependence of LLs on the magnetic field and level index, even at low defect concentrations.

\section{ACKNOWLEDGMENTS}

We thank J. M. Pereira Jr. for useful discussions. The support by the Stichting Fundamenteel Onderzoek der Materie (FOM) and the Netherlands National Computing Facilities foundation (NCF) are acknowledged. We are grateful for financial support from the European Research Council Advanced Grant program (contract 338957). The research has also received funding from the European Union Seventh Framework Programme under Grant Agreement No. 604391 Graphene Flagship.

\section{APPENDIX}

The unit cell of single-layer BP contains four atoms, and the TB Hamiltonian can be represented as $[8,48]$

$$
H=\left(\begin{array}{cccc}
0 & t_{2} \varphi_{2}+t_{5} \varphi_{5} & t_{4} \varphi_{4} & t_{1} \varphi_{1}+t_{3} \varphi_{3} \\
t_{2} \varphi_{2}^{*}+t_{5} \varphi_{5}^{*} & 0 & t_{1} \varphi_{1}^{*}+t_{3} \varphi_{3}^{*} & t_{4} \varphi_{4}^{*} \\
t_{4} \varphi_{4}^{*} & t_{1} \varphi_{1}+t_{3} \varphi_{3} & 0 & t_{2} \varphi_{2}+t_{5} \varphi_{5} \\
t_{1} \varphi_{1}^{*}+t_{3} \varphi_{3}^{*} & t_{4} \varphi_{4} & t_{2} \varphi_{2}^{*}+t_{5} \varphi_{5}^{*} & 0
\end{array}\right),
$$


where the phase terms $\varphi_{i}$ are defined as

$$
\begin{gathered}
\varphi_{1}=2 e^{i d k_{y}} \cos \left(c k_{x}\right), \\
\varphi_{2}=e^{-i b k_{y}}, \\
\varphi_{3}=2 e^{-i(2 b+d) k_{y}} \cos \left(c k_{x}\right), \\
\varphi_{4}=4 \cos \left(c k_{x}\right) \cos \left((b+d) k_{y}\right), \\
\varphi_{5}=e^{i(b+2 d) k_{y}},
\end{gathered}
$$

and the constants $c=a \sin \theta, d=a \cos \theta, \theta=48.395^{\circ}, a \approx$ $2.216 \AA$, and $b \approx 0.716 \AA$ are the atomic distance of two nearest neighbors projected onto the surface plane. The four eigenvalues of the TB Hamiltonian matrix can be represented as

$$
\begin{gathered}
E_{1}\left(k_{x}, k_{y}\right)=A\left(k_{x}, k_{y}\right)-B\left(k_{x}, k_{y}\right), \\
E_{2}\left(k_{x}, k_{y}\right)=A\left(k_{x}, k_{y}\right)+B\left(k_{x}, k_{y}\right), \\
E_{3}\left(k_{x}, k_{y}\right)=-A\left(k_{x}, k_{y}\right)-C\left(k_{x}, k_{y}\right), \\
E_{4}\left(k_{x}, k_{y}\right)=-A\left(k_{x}, k_{y}\right)+C\left(k_{x}, k_{y}\right),
\end{gathered}
$$

where $A\left(k_{x}, k_{y}\right), B\left(k_{x}, k_{y}\right)$, and $C\left(k_{x}, k_{y}\right)$ are

$$
\begin{aligned}
A\left(k_{x}, k_{y}\right)= & 4 t_{4} \cos \left(c k_{x}\right) \cos \left((b+d) k_{y}\right), \\
B\left(k_{x}, k_{y}\right)= & {\left[2 \cos \left(2 c k_{x}\right)\left\{2 t_{1} t_{3} \cos \left[2(b+d) k_{y}\right]+t_{1}^{2}+t_{3}^{2}\right\}+4 \cos \left(c k_{x}\right)\left[t_{2}\left(t_{1}+t_{3}\right)+t_{1} t_{5}\right] \cos \left((b+d) k_{y}\right)\right.} \\
& \left.+t_{3} t_{5} \cos \left(3(b+d) k_{y}\right)+2\left(2 t_{1} t_{3}+t_{2} t_{5}\right) \cos \left(2(b+d) k_{y}\right)+2 t_{1}^{2}+t_{2}^{2}+2 t_{3}^{2}+t_{5}^{2}\right]^{1 / 2}, \\
C\left(k_{x}, k_{y}\right)= & {\left[-4 t_{3} t_{5} \cos \left(c k_{x}\right) \cos \left(3(b+d) k_{y}\right)+2 \cos \left(2(b+d) k_{y}\right)\left[2 t_{1} t_{3} \cos \left(2 c k_{x}\right)+2 t_{1} t_{3}+t_{2} t_{5}\right]\right.} \\
& \left.-4\left[t_{2} t_{3}+t_{1}\left(t_{2}+t_{5}\right)\right] \cos \left(c k_{x}\right) \cos \left((b+d) k_{y}\right)+2\left(t_{1}^{2}+t_{3}^{2}\right) \cos \left(2 c k_{x}\right)+2 t_{1}^{2}+t_{2}^{2}+2 t_{3}^{2}+t_{5}^{2}\right]^{1 / 2} .
\end{aligned}
$$

$E_{1}\left(k_{x}, k_{y}\right)$ and $E_{2}\left(k_{x}, k_{y}\right)$ are the lowest valence $\left(E^{v}\right)$ and conduction $\left(E^{c}\right)$ bands fit to $G W$ calculations and plotted in Fig. 1.

The Fermi velocity can be obtained via $v_{\alpha}=\frac{1}{\hbar} \frac{\partial E}{\partial k_{\alpha}}$, and the electron and hole velocities along the armchair $(Y)$ and zigzag $(X)$ directions are

$$
\begin{aligned}
& v_{x}^{v}=A_{1}-B_{1} / D, \\
& v_{y}^{v}=A_{2}-B_{2} / D, \\
& v_{x}^{c}=A_{1}+B_{1} / D, \\
& v_{y}^{c}=A_{2}+B_{2} / D,
\end{aligned}
$$

where

$$
\begin{aligned}
A_{1}= & -4 c t_{4} \sin \left(c k_{x}\right) \cos \left((b+d) k_{y}\right), \\
A_{2}= & -4 t_{4}(b+d) \cos \left(c k_{x}\right) \sin \left((b+d) k_{y}\right), \\
B_{1}= & -4 c \sin \left(2 c k_{x}\right)\left[2 t_{3} t_{1} \cos \left(2(b+d) k_{y}\right)+t_{1}^{2}+t_{3}^{2}\right] \\
& -4 c \sin \left(c k_{x}\right)\left\{t_{3} t_{5} \cos \left(3(b+d) k_{y}\right)+\left[t_{2}\left(t_{1}+t_{3}\right)+t_{1} t_{5}\right] \cos \left((b+d) k_{y}\right)\right\}, \\
B_{2}= & -8 t_{1} t_{3}(b+d) \cos \left(2 c k_{x}\right) \sin \left(2(b+d) k_{y}\right)-4\left(2 t_{1} t_{3}+t_{2} t_{5}\right)(b+d) \sin \left(2(b+d) k_{y}\right) \\
& +4 \cos \left(c k_{x}\right)\left\{-3 t_{3} t_{5}(b+d) \sin \left(3(b+d) k_{y}\right)-\left[t_{2}\left(t_{1}+t_{3}\right)+t_{1} t_{5}\right](b+d) \sin \left((b+d) k_{y}\right)\right\}, \\
D= & 2\left[2 \cos \left(2 c k_{x}\right)\left[2 t_{3} t_{1} \cos \left(2(b+d) k_{y}\right)+t_{1}^{2}+t_{3}^{2}\right]\right. \\
& +4 \cos \left(c k_{x}\right)\left\{t_{3} t_{5} \cos \left(3(b+d) k_{y}\right)+\left[t_{2}\left(t_{1}+t_{3}\right)+t_{1} t_{5}\right] \cos \left((b+d) k_{y}\right)\right\} \\
& \left.+2\left(2 t_{1} t_{3}+t_{2} t_{5}\right) \cos \left(2(b+d) k_{y}\right)+2 t_{1}^{2}+t_{2}^{2}+2 t_{3}^{2}+t_{5}^{2}\right]^{1 / 2} .
\end{aligned}
$$

The calculation of effective mass is straightforward via $1 / m_{\alpha}=\frac{\partial^{2} E}{\partial k_{\alpha}^{2}} / \hbar^{2}$, and for $k_{x}=k_{y}=0$, we have

$$
\begin{gathered}
m_{x}^{v}=-4 c^{2} t_{4}-A_{3} / F, \\
m_{y}^{v}=-4 t_{4}(b+d)^{2}-A_{4} / F, \\
m_{x}^{c}=-4 c^{2} t_{4}+A_{3} / F, \\
m_{y}^{c}=-4 t_{4}(b+d)^{2}+A_{4} / F,
\end{gathered}
$$


where

$$
\begin{aligned}
& A_{3}=-8 c^{2}\left(t_{1}^{2}+2 t_{3} t_{1}+t_{3}^{2}\right)-4 c^{2}\left[t_{2}\left(t_{1}+t_{3}\right)+t_{1} t_{5}+t_{3} t_{5}\right] \\
& A_{4}=-16 t_{1} t_{3}(b+d)^{2}-8\left(2 t_{1} t_{3}+t_{2} t_{5}\right)(b+d)^{2}+4\left\{\left[t_{2}\left(t_{1}+t_{3}\right)+t_{1} t_{5}\right](-b-d)(b+d)-9 t_{3} t_{5}(b+d)^{2}\right\}, \\
& F=2 \sqrt{2 t_{1}^{2}+t_{2}^{2}+2 t_{3}^{2}+t_{5}^{2}+2\left(t_{1}^{2}+2 t_{3} t_{1}+t_{3}^{2}\right)+2\left(2 t_{1} t_{3}+t_{2} t_{5}\right)+4\left(t_{2}\left(t_{1}+t_{3}\right)+t_{1} t_{5}+t_{3} t_{5}\right)} .
\end{aligned}
$$

By using the parameters of single-layer BP, the effective masses at $\Gamma$ point are

$$
\begin{gathered}
m_{x}^{v}=-1.143 m_{e}, \\
m_{y}^{v}=-0.184 m_{e}, \\
m_{x}^{c}=0.849 m_{e}, \\
m_{y}^{c}=0.167 m_{e} .
\end{gathered}
$$

[1] L. Li, Y. Yu, G. J. Ye, Q. Ge, X. Ou, H. Wu, D. Feng, X. H. Chen, and Y. Zhang, Nat. Nanotech. 9, 372 (2014).

[2] H. Liu, A. T. Neal, Z. Zhu, Z. Luo, X. Xu, D. Tománek, and P. D. Ye, ACS Nano 8, 4033 (2014).

[3] F. Xia, H. Wang, and Y. Jia, Nat. Commun. 5, 4458 (2014).

[4] S. P. Koenig, R. A. Doganov, H. Schmidt, A. H. Castro Neto, and B. Özyilmaz, Appl. Phys. Lett. 104, 103106 (2014).

[5] A. Castellanos-Gomez et al., 2D Mater. 1, 025001 (2014).

[6] L. Li et al., arXiv:1411.6572.

[7] J. Qiao, X. Kong, Z.-X. Hu, F. Yang, and W. Ji, Nat. Commun. 5, 4475 (2014).

[8] A. N. Rudenko and M. I. Katsnelson, Phys. Rev. B 89, 201408 (2014).

[9] V. Tran, R. Soklaski, Y. Liang, and L. Yang, Phys. Rev. B 89, 235319 (2014).

[10] J. Guan, Z. Zhu, and D. Tománek, Phys. Rev. Lett. 113, 046804 (2014).

[11] X. Peng, Q. Wei, and A. Copple, Phys. Rev. B 90, 085402 (2014).

[12] D. Çakır, H. Sahin, and F. M. Peeters, arXiv:1411.1344.

[13] T. Low, A. S. Rodin, A. Carvalho, Y. Jiang, H. Wang, F. Xia, and A. H. Castro Neto, Phys. Rev. B 90, 075434 (2014).

[14] T. Low, M. Engel, M. Steiner, and P. Avouris, Phys. Rev. B 90, 081408 (2014).

[15] P. Li and I. Appelbaum, Phys. Rev. B 90, 115439 (2014).

[16] A. Favron, E. Gaufrès, F. Fossard, P. L. Lévesque, A. PhaneufL'Heureux, N. Y-W. Tang, A. Loiseau, R. Leonelli, S. Francoeur, and R. Martel, arXiv:1408.0345.

[17] J. D. Wood, S. A. Wells, D. Jariwala, K.-S. Chen, E. Cho, V. K. Sangwan, X. Liu, L. J. Lauhon, T. J. Marks, and M. C. Hersam, Nano Lett. 14, 6964 (2014).

[18] J. O. Island, G. A. Steele, H. S. J. van der Zant, and A. Castellanos-Gomez, 2D Mater. 2, 011002 (2015).

[19] A. H. C. Neto, F. Guinea, N. M. R. Peres, K. S. Novoselov, and A. K. Geim, Rev. Mod. Phys. 81, 109 (2009).

[20] S. Das Sarma, S. Adam, E. H. Hwang, and E. Rossi, Rev. Mod. Phys. 83, 407 (2011).

[21] M. I. Katsnelson, Graphene: Carbon in Two Dimensions (Cambridge University Press, Cambridge, 2012).
[22] T. O. Wehling, S. Yuan, A. I. Lichtenstein, A. K. Geim, and M. I. Katsnelson, Phys. Rev. Lett. 105, 056802 (2010).

[23] S. Yuan, H. De Raedt, and M. I. Katsnelson, Phys. Rev. B 82, 115448 (2010).

[24] S. Yuan, H. De Raedt, and M. I. Katsnelson, Phys. Rev. B 82, 235409 (2010).

[25] H. Qiu et al., Nat. Commun. 4, 2642 (2013).

[26] S. Yuan, R. Roldán, M. I. Katsnelson, and F. Guinea, Phys. Rev. B 90, 041402 (2014).

[27] Y. Liu, F. Xu, Z. Zhang, E. S. Penev, and B. I. Yakobson, Nano Lett. 14, 6782 (2014).

[28] W. Hu and J. Yang, arXiv:1411.6986.

[29] A. Ziletti, A. Carvalho, D. K. Campbell, D. F. Coker, and A. H. Castro Neto, Phys. Rev. Lett. 114, 046801 (2015).

[30] R. Zhang, B. Li, and J. Yang, arXiv:1409.7190.

[31] V. V. Kulish, O. I. Malyi, C. Persson, and P. Wu, Phys. Chem. Chem. Phys. 17, 992 (2015).

[32] J. Martin, N. Akerman, G. Ulbricht, T. Lohmann, J. H. Smet, K. von Klitzing, and A. Yacoby, Nat. Phys. 4, 144 (2007).

[33] E. H. Hwang, S. Adam, and S. Das Sarma, Phys. Rev. Lett. 98, 186806 (2007).

[34] Y. Zhang, V. W. Brar, C. Girit, A. Zettl, and M. F. Crommie, Nat. Phys. 5, 722 (2009).

[35] A. N. Rudenko, F. J. Keil, M. I. Katsnelson, and A. I. Lichtenstein, Phys. Rev. B 84, 085438 (2011).

[36] M. Gibertini, A. Tomadin, M. Polini, A. Fasolino, and M. I. Katsnelson, Phys. Rev. B 81, 125437 (2010).

[37] M. Gibertini, A. Tomadin, F. Guinea, M. I. Katsnelson, and M. Polini, Phys. Rev. B 85, 201405 (2012).

[38] S. Yuan, R. Roldán, H. De Raedt, and M. I. Katsnelson, Phys. Rev. B 84, 195418 (2011).

[39] S. Yuan, T. O. Wehling, A. I. Lichtenstein, and M. I. Katsnelson, Phys. Rev. Lett. 109, 156601 (2012).

[40] A. Hams and H. De Raedt, Phys. Rev. E 62, 4365 (2000).

[41] J. D. Jackson, Classical Electrodynamics, 3rd ed. (Wiley, New York, 1999).

[42] A. Ishihara, Statistical Physics (Academic Press, New York, 1971). 
[43] C. H. Lewenkopf, E. R. Mucciolo, and A. H. Castro Neto, Phys. Rev. B 77, 081410 (2008).

[44] J. Ziman, Models of Disorder: The Theoretical Physics of Homogeneously Disordered Systems (Cambridge University Press, Cambridge, 1979).

[45] N. Mott and E. Davis, Electronic Processes in Non-Crystalline Materials (Oxford University Press, Oxford, 2012).
[46] T. Wehling, M. Katsnelson, and A. Lichtenstein, Chem. Phys. Lett. 476, 125 (2009).

[47] A. S. Rodin, A. Carvalho, and A. H. Castro Neto, Phys. Rev. Lett. 112, 176801 (2014).

[48] M. Ezawa, New J. Phys. 16, 115004 (2014).

[49] X. Y. Zhou, R. Zhang, J. P. Sun, Y. L. Zou, D. Zhang, W. K. Lou, F. Cheng, G. H. Zhou, F. Zhai, and K. Chang, arXiv:1411.4275. 